\title{
TEORIA E MÉTODOS NA ANÁLISE CERÂMICA EM ARQUEOLOGIA
}

O estudo da cerâmica ocupa, certamente, uma posição expressiva na disciplina arqueológica. Isto se deve, em primeiro lugar, à universalidade deste tipo de vestígio: além de a cerâmica ser encontrada em contextos arqueológicos de praticamente todo o mundo, possui uma história bastante recuada no tempo. Os primeiros objetos identificados correspondem a figuras de argila, datadas em $26.000 \mathrm{BP}$ e encontradas em grande número no sítio Dolni Vestonice, no leste europeu (Vandiver et al. 1989). Já a mais antiga indústria de vasilhas foi datada em $12.700 \mathrm{BP}$, correspondendo à cerâmica Jomon, no sudeste do Japão (Aikens 1995).

A partir daí, a manufatura da cerâmica emergiu de forma independente em diversas áreas do mundo, e seu conhecimento foi rapidamente espalhado. Isto faz com que constitua, muitas vezes, o principal vestígio no estudo de sociedades que se desenvolveram nos últimos 10.000 anos.

Por outro lado, graças às suas propriedades físicas, os vestígios cerâmicos têm grande durabilidade, permitindo ao arqueólogo recuperar uma porção expressiva dessa indústria. Além disto, os fragmentos cerâmicos exercem, seguramente, uma atração bem menor entre colecionadores (mais inclinados a coletar pontas projéteis lascadas ou machadinhas polidas), fornecendo contextos de pesquisa menos alterados.

Finalmente, devemos indicar o potencial interpretativo que a cerâmica apresenta. Se, por um lado, grande parte dos utensílios cerâmicos está relacionada a funções cotidianas coletivas (como o preparo de alimentos, cozimento e estocagem), pode estar igualmente vinculada a usos específicos e restritos a determinados grupos de indivíduos (como o comércio e/ou troca de bens, rituais, etc...). Assim, uma vez que a cerâmica pode estar relaciona-

(*) Museu de Arqueologia e Etnologia da Universidade de São Paulo. da a contextos distintos, permitiria acesso a fenômenos culturais diversificados.

Todos estes fatores fizeram com que o estudo da cerâmica estivesse sempre presente, acompanhando o caminho trilhado pela Arqueologia enquanto disciplina. Seu histórico já foi apresentado a partir de diferentes classificações: Shepard (1956) dividiu-o em três fases sem, entretanto, estabelecer referências cronológicas. São elas: o estudo da vasilha enquanto objeto cultural; o estudo dos fragmentos na obtenção de sequiências estratigráficas; e o estudo da tecnologia cerâmica como via de acesso ao próprio ceramista. Matson (1984) e Rice (1988), baseados na Arqueologia americana e apoiados nas fases definidas por Willey e Sabloff (1974), estabeleceram: o período histórico classificatório (19141960) e o período explanatório (de 1960 em diante). Van der Leeuw (1984) definiu três fases: a tipológica (até 1965); os "três níveis de pesquisa" (1965-1980); e o estudo dos elementos culturais (a partir de 1980). Por fim, Orton, Tyers \& Vinces (1995) propõem uma divisão em outras três fases: a artística/histórica, a tipológica e a fase contextual. $\mathrm{O}$ breve histórico que fornecemos a seguir se baseia no trabalho destes últimos, já que apresentam, ao nosso ver, uma classificação mais abrangente e atual.

Segundo os autores, cada fase remete a uma escala distinta de estudo: enquanto a fase artística/ histórica reflete sobre a vasilha como um todo, a fase tipológica se baseia nos fragmentos. Por fim, a fase contextual reuniria trabalhos com enfoques bastante distintos, passando do detalhe microscópico de fabricação da cerâmica à comparação de conjuntos de artefatos provenientes de sítios diversos. Embora, de um modo geral, estas fases tenham sucedido ao longo do tempo, apresentam muitas vezes desenvolvimentos paralelos, motivados por questões específicas que cada pesquisa apresenta.

A fase artística/histórica, cujos primeiros trabalhos remontam pelo menos ao século $\mathrm{XV}$, tem 
como origem a descrição da cerâmica tecnologicamente mais elaborada tanto da Europa como da Ásia, com destaque para as vasilhas etruscas, gregas e romanas (Sklenár 1983; Stow 1603; van Mellen 1679; Browne 1658; Groevius e Groonovius 1694, entre outros, citados por Orton et alii 1995: 5).

Os trabalhos tratam, em grande parte, de vasilhas associadas a enterramentos. Discorrendo sobre peças individuais ou sobre coleções, a ênfase recaía nas técnicas de fabricação e nos aspectos decorativos, muitas vezes trazendo interpretações de cenas clássicas. No final do século XVIII e início do XIX expressa-se um interesse mais geral pela cerâmica, relacionada a períodos e localidades variadas, embora predominem ainda estudos voltados às peças mais elaboradas.

O estudo da cerâmica doméstica européia pósclássica só se desenvolveu, de forma sistemática, a partir do século XX. Quanto ao estudo da cerâmica Oriental (ainda predominantemente chinesa e japonesa), após uma verdadeira era de colecionadores, as atenções se voltaram a questões históricas, abordando, por exemplo, temas como o comércio de bens (Julien 1856; Noritané 1876; Hirth 1888, citados por Orton et alii 1995: 8).

Nos EUA, o estudo da cerâmica teve início no final do século XVIII e princípio do XIX, igualmente relacionado a investigações de monumentos (como por exemplo os trabalhos de Squier e Davis 1848; Schoolcraft 1847). A fundação do Bureau of American Ethnology constituiu um avanço significativo nas investigações. Estudos paralelos começaram a ser desenvolvidos no restante da América, como o trabalho de Waldek (1838) na América Central, e o de Falbe (1843) na América do Sul (citados por Orton et alii 1995: 8).

A fase tipológica teria surgido da necessidade de classificar a grande quantidade de material coletado em escavações na França, Alemanha e Bretanha, no final do século XIX (Rhodes 1979, referindo-se a Smith 1854; Plique 1887; Cochet 1860; Solon 1910, entre outros, citados por Orton et alii 1995: 9). Inicia-se neste período a relação da cerâmica a seqüências estratigráficas (vide o clássico trabalho de Petrie, 1891, em Lachish, na Palestina). Nos Estados Unidos, isto se deu com as escavações de Kidder $(1924,1931)$ em Pecos, propondo uma integração entre estratigrafia, levantamentos regionais e cerâmica. Segundo Orton et alii (1995:9), este trabalho constituiu um modelo para os métodos arqueológicos desenvolvidos nos anos 60 .
Durante a fase tipológica, os estudos se direcionaram, por um lado, para a distribuição vertical (ou cronológica) da cerâmica, a partir da sequiência estratigráfica dos achados; por outro lado, o enfoque horizontal, voltado à análise de distribuição da cerâmica no espaço, contribuiu para estabelecer seqüências regionais, bem como definir áreas culturais (Childe 1929).

O principal instrumento metodológico foi a seriação, criada como uma maneira de ordenar os vestígios através da presença ou ausência de artefatos (ou atributos)-tipo. A aplicação do conceito em coleções de superfície foi feita por Spier (1917), ao mesmo tempo em que se implantava a idéia de que a proporção de ocorrência dos tipos na estratigrafia obedecia a padrões (Nelson 1916). Sugeriuse, então, que os padrões teriam um significado cultural, e o método da seriação passa a ser utilizado para criar cronologias culturais, baseadas em cálculos matemáticos (Ford 1962).

$\mathrm{Na}$ arqueologia americana, o conceito do "tipo" adquire grande importância. Uma vez criado, ele poderia ser ordenado de acordo com idéias de "desenvolvimento" e utilizado para demonstrar sequiências cronológicas, dentro de um raciocínio circular (Orton et alii 1995:11). Gifford (1960: 341) definiu tipo como "a specific kind of pottery embodying a unique combination of recognizable distinct attributes". Segundo os pressupostos desta escola, os tipos (sejam cerâmicos ou líticos) permitiriam identificar relacionamentos históricos entre culturas.

A classificação em tipos logo se mostrou, entretanto, insuficiente, considerando a grande diversidade de variáveis que a cerâmica apresenta. Isto levou à adoção do conceito de "variedades" (Krieger 1944; Gifford 1960) ou de outras classificações como sequiências, séries, sistemas cerâmicos ou modos (Orton et alii 1995:12; Rouse 1960; Whallon 1972).

A necessidade de fornecer descrições detalhadas dos tipos cerâmicos fez proliferar as publicações voltadas à classificação. Incluem-se aqui, entre outros, os estudos de fonte de matéria prima (Peacock 1977), textura da cerâmica (Guthe 1927; Byers 1937) e antiplástico (Shepard 1964). Uma análise comparativa entre estes diferentes trabalhos pode ser obtida em Robinson (1979).

Nesta fase tipológica foram criados os principais fundamentos para o estudo da cerâmica, a maioria deles ainda hoje utilizados. Destacam-se 
aqui as análises tecnológicas (principalmente petrográficas e químicas) e os estudos de fontes de argila.

Ainda na década de 50, deu-se início à denominada fase contextual que, segundo Orton et alli (1995: 13), estaria relacionada à publicação da obra Ceramics for the Archaeologist, de A. Shepard, em 1956. O livro articula, pela primeira vez, análises sobre cronologia, desenvolvimento tecnológico e distribuição da cerâmica. Apresenta, ainda, uma série de contribuições para a análise da cerâmica, tanto práticas quanto teóricas. Neste último caso, discute detalhadamente o uso e limitações do conceito de "tipo", bem como sobre sua suposta relação com entidades culturais.

Depois do trabalho de Shepard, o estudo da cerâmica se projetou em uma grande variedade de direções, resultando em trabalhos com objetivos, abordagens e resultados notadamente diversos. Como pontos comuns a estes estudos, podem ser citados, principalmente:

- uma grande resistência ao conceito de considerar os fragmentos cerâmicos enquanto tipos-fósseis, principalmente quando a evolução das técnicas de datação radiocarbônica substituíram, com larga vantagem, o uso das tipologias para estabelecer cronologias culturais;

- uma continuidade no desenvolvimento de técnicas cada vez mais específicas e precisas para a análise da cerâmica, nos campos da física, química e matemática (esta última através da aplicação de diferentes programas estatísticos);

- e, por fim, a percepção de que diferentes processos de formação de sítio (envolvendo descarte, remanejamentos internos ainda durante a ocupação do sítio e toda a gama de alterações posteriores) necessitam ser considerados na análise da cerâmica, deixando de lado a correlação simplista, muitas vezes adotada, entre vasilha em uso e fragmentos escavados.

Os sítios passam a não ser mais tratados individualmente, mas sim em conjunto, a partir de estudos comparativos. Isto levou à necessidade de uniformizar procedimentos de análise e nomenclaturas, apresentados por manuais como o de Rye (1981) e de Rice (1987), publicados nos EUA, e como de Balfet et al. (1989), Blake and Davey (1983) e Young (1980), publicados na Europa.

Por outro lado, a pesquisa arqueológica passou a reconhecer o valor dos estudos etnográficos, tanto no desenvolvimento de suas análises e interpreta- ções, como para a construção de modelos. Já no início do século XX surgem os primeiros trabalhos que consideram os processos de produção da cerâmica (Linné 1925; Bunzel 1929), bem como uma tentativa inicial em relacionar a etnografia com a arqueologia (Franchet 1911). Depois disto, foram inúmeros os estudos que se dedicaram ao tema.

A Etnoarqueologia, área de pesquisa que se consolidou mais recentemente, propõe desenvolver estudos arqueológicos em sociedades contemporâneas, desenvolvendo e testando hipóteses sobre a relação entre cultura material e comportamento, e sugerindo relacionamentos similares no passado. Desta forma, é capaz de apresentar contribuições sobre temas diversificados e, muitas vezes, de difícil tratamento na Arqueologia, como padrões de transmissão de conhecimento cerâmico, posição social do ceramista, sistemas de distribuição e uso dos artefatos, dados sobre a vida útil da cerâmica, padrões de quebra e práticas de descarte, entre outros (Hendrickson \& McDonald 1983; Hodder 1982, 1989; Kent 1984; Kramer 1979; Layton 1994; Longagre 1991; Arnold 1991; Skibo 1992).

De todas as fases por que o estudo da cerâmica arqueológica passou, desde a fase artística/histórica, tipológia e contextual, o grande desafio dos pesquisadores foi, e ainda é, compreender até que ponto a cerâmica se articula com aspectos não materiais da cultura, envolvendo esferas políticas, sociais, econômicas, ideológicas ou históricas. Permanece, portanto, a indagação: qual o significado da cerâmica para o estudo de sociedades humanas, ao longo. do tempo?

Algumas pesquisas apontam um panorama desanimador. É o caso, por exemplo, do trabalho de Tschopik (1950), que observou uma variação muito pequena da cerâmica Aymara (identificada na bacia do Titicaca, Peru) desde o período précolombiano até o presente, mesmo que seus portadores tenham passado pela dominação Inca, pela conquista espanhola e por 400 anos de maciça aculturação. Outro exemplo similar é fornecido por Charlton (1976), indicando que as mudanças na cerâmica do vale do México frente à dominação espanhola foram extremamente suaves e graduais, tendo, inclusive, se iniciado somente 150 anos após a conquista.

De fato, nem sempre a relação entre a variabilidade da cerâmica e questões sobre organização política, social, econômica, ideológica e suas mudanças ao longo do tempo, são óbvias e diretas. Em 
muitos casos, outras classes de cultura material seriam mais adequadas do que a cerâmica para discutir estas questões (como por exemplo o vestuário, ou a pintura corporal). Todavia, geralmente é com a cerâmica que o arqueólogo depara. Embora ela não se preste para equacionar todas as questões, um trabalho cuidadoso, a partir de pressupostos teóricos e metodológicos bem definidos, certamente permitirá ir muito mais além do que simplesmente agrupar atributos no tempo e no espaço.

No que se refere à metodologia de análise, embora tenham sido apresentados grandes avanços com o uso de tecnologias sofisticadas, os paradigmas analíticos usualmente adotados limitam, de forma significativa, as questões que podem ser respondidas. Grande parte das análises ainda hoje desenvolvidas dão ênfase à classificação do material, onde os fragmentos cerâmicos constituem a base de estudo, novamente partidos em atributos e subatributos de análise. De fato, o fragmento cerâmico, enquanto elemento isolado, não constitui uma unidade cultural de comportamento. Somente através do estudo da produção, uso e descarte da vasilha enquanto artefato é que poderão ser desenvolvidos estudos propriamente antropológicos, ampliando as discussões e o alcance da pesquisa arqueológica.

Dentro desta discussão, uma das grandes contribuições fornecidas pela literatura é a revisão crítica sobre a classificação da cerâmica. A classificação de artefatos, ou a divisão do material arqueológico em categorias segundo suas semelhanças ou diferenças, constitui uma parte essencial em qualquer estudo arqueológico. Uma vez que lidamos com sociedades extintas, não temos acesso direto a como seus indivíduos interpretavam ou classificavam a cultura material. Assim, a classificação de indústrias arqueológicas recai sobre uma variedade de fatores, incluindo matéria prima, distribuição temporal e espacial, bem como padrões de similaridades formais, morfológicas e decorativas. Contamos hoje com inúmeros debates sobre a natureza da classificação arqueológica, onde se destaca uma vasta literatura sobre métodos quantitativos, definindo e mensurando de forma explícita os atributos da cerâmica, e utilizando diferentes testes estatísticos para examinar suas relações e distribuição.

Por outro lado, os próprios procedimentos de coleta do material, em campo, muitas vezes ainda são destituídos de problemas específicos de pesquisa e assim, conseqüentemente, não irão suportar o desenvolvimento de análises mais aprofundadas. Como tantos já alertaram, e por tantas vezes, volto a mencionar que o alcance de nossas conclusões é definido, antes de mais nada, pelo alcance de nossos problemas de pesquisa. Sem dúvida, a cerâmica se mostrará mais apropriada para responder questões sobre o passado se trabalharmos com amostras criteriosamente coletadas e documentadas, e quando for associada a seu respectivo contexto cultural.

É notável que a última década tenha apresentado uma enorme proliferação na literatura sobre cerâmica arqueológica. Isto se deve, por um lado, ao próprio potencial da cerâmica na pesquisa arqueológica; por outro lado, reflete nossa crescente habilidade em colocar e responder questões sobre o passado. Dentro deste contexto, mencionamos abaixo algumas linhas de pesquisa que têm recebido maior atenção nos últimos anos e que forneceram, conseqüentemente, interessantes contribuições para o avanço da análise cerâmica em Arqueologia.

Em primeiro lugar, devemos citar os estudos que se voltam aos aspectos tecnológicos, valendose de recursos cada vez mais sofisticados. Podemos citar aqui, entre outros, os trabalhos de Stoltman (1989), Neff (1992, 1993), Cogswell et alii 1998; Giardino et alii 1998; e de Rice 1987. Tecnologia cerâmica chegou inclusive a constituir o tema do volume 21 da revista World Archaeology (1989), reunindo onze artigos sobre o assunto. Contamos ainda com trabalhos que não apenas discorrem sobre métodos de análise e questões tecnológicas, mas procuram discutir os resultados obtidos dentro de problemas arqueológicos mais amplos. Incluemse aqui trabalhos como de Stoltman (1991), que utiliza análises petrográficas na cerâmica para discutir aspectos de interação cultural, e o trabalho de Pärssinan e Siiriäinen (1997) que utiliza aspectos tecnológicos e cronológicos da cerâmica para discutir processos históricos da expansão Inca.

A questão do uso, produção e distribuição da cerâmica, envolvendo problemas sobre sistemas de troca e aspectos econômicos, foi desenvolvida por autores como Pool \& Bey (1992). Estudos de caso são oferecidos, entre outros, por Strazicich (1998), que discute aspectos de economia regional através da produção cerâmica e, mais especificamente, através da identificação de fontes de argila diversificadas; por Osborne (1996), que analisa aspectos de comércio de artefatos cerâmicos na Grécia; por Moore (1995), que avalia o impacto na economia 
do Oeste Asiático através da introdução da cerâmica; por Gebauer (1996), que discute a relação entre produção cerâmica e introdução da agricultura na Escandinávia; e por Crown \& Wills (1996), que apresentam um estudo sobre intensificação da economia e aparecimento de vasilhas cerâmicas no sudoeste americano.

Já a questão da cerâmica e organização social e política foi abordada por trabalhos como o de Donnan (1997), que discute o problema da divisão do trabalho e uso hierarquizado da cerâmica na costa norte do Peru; os trabalhos de Mc Govern e Notis (1989), Hegmon (1997) e Hoopes (1995), que discutem aspectos de interação cultural a partir da análise cerâmica; de Sassaman (1996) que avalia aspectos sociais derivados do uso de tecnologias de cozimento tradicionais e inovadoras, no sudeste americano; além de uma série de estudos de caso sintetizados por Sinopoli (1991: 119-160).

Por fim, a questão da cerâmica em estudos de continuidade e mudança cultural foi discutida por autores como Bey III et alii (1997), que propõem uma associação entre presença de cerâmica e contextos arquitetônicos específicos nas terras baixas Maias, como indicadores da transição entre o período clássico e pós-clássico; Armit \& Finlayson (1996), que discutem o papel da cerâmica na transição entre os períodos Mesolítico e Neolítico europeu; e, no Brasil, por trabalhos voltados à ocupação de grupos ceramistas na região Centro-Oeste, desenvolvidos por Wüst (1990) e por Robrahn-González (1996).

Como se vê, o estudo da cerâmica tem permitido avançar discussões sobre problemas arqueológicos diversificados. Isto se deve, sem dúvida, à versatilidade deste tipo de evidência, possibilitando a proposição de novas técnicas e/ou abordagens e, desta forma, ampliando seu potencial interpretativo. Certamente a cerâmica continuará sendo explorada no constante desenvolvimento de nossa disciplina, cabendo a nós, arqueólogos, alargar o leque de questões que pode ser por ela respondido.

\section{Referências bibliográficas}

AIKENS, C.M.

1995 The Jomon Pottery of Early Japan. W.K.Barnett and J.W.Hoopes (Eds.) The Emergence of Pottery - technology and innovation in Ancient Societies. Washington, London, Smithsonian Institution Press: 11-22.

ARMIT, I.; FINLAYSON, B.

1996 Social Strategies and Economic Change. W.K. Barnett; J.W. Hoopes (Eds.) The emergence of Pottery. Washington, London, Smithsonian Institution Press: 267-276.

ARNOLD, D.E.

1985 Ceramic Theory and cultural process. Cambridge, Cambridge University Press.

1991 Domestic Ceramic Production and spatial organization: a mexican case study in Ethnoarchaeology. Cambridge, Cambridge Univ. Press.

BALFET, H.; FAUVET BERTHELOT, M.F.; MOZON,S.

1989 Lexique et typologie des poteries: pour la normalisation de la description des poteries. $\mathrm{Pa}-$ ris, Presses du CNRS.

BEY III, G.J.; HANSON, C.A.; RINGLE, W.M.

1997 Classic to Postclassic at Ek Balam, Yucatan: Architectural and Cêramic evidence for defining the transition. Latin American Antiquity, 8 (3): 237-254.

BLAKE, H.; DAVEY, P.

1983 Guidelines for the processing and publication of medieval pottery from excavations. Directorate of Ancient Monuments and Historic Buildings Occasional Paper, 5, Londres.
BRAUN, D.

1983 Pots as tools. A.S.Keene; J.A.Moore (Eds.) Archaeological Hammers and Theories. Nova Iorque, Academic Press: 107-134.

BROWNE, T.

1658 Hydriotaphia. Urne burial. Londres.

BYERS, D.S.

1937 On standards for texture in pottery, American Antiquity, 3: 76-7.

CHARLTON, T.H.

1976 Contemporary central Mexican ceramics: a view from the past. Man (n.s.), 11: 517-25.

CHILDE, V.G.

1929 The Danube in Prehistory. Oxford, Clarendon Press.

COCHET, A

1860 Archeologie céramique et sépulchrale: ou l'art de classer les sépultures anciennes à l'aide de la Céramique. Paris.

COGSWELL, J.W.; NEFF, H.; GLASCOCK, M.

1998 Analysis of Shell-tempered pottery replicates: implications for Provenance Studies. American Antiquity, 63 (1): 63-72.

CROWN,P.L.; WILLS,W.H.

1996 Economic Intensification and the origin of Ceramic Containers in the American Southwest. W.K. Barnett; J.W. Hoopes (Eds.) The emergence of Pottery. Washington, Smithsonian Institution Press: 241-256.

DEETZ, J.

1965 The dynamics of stylistic change in Arikara ceramics. Urbana Univ. of Illinois Press. 
DONNAN, C.B.

1997 A Chimu-Inka Ceramic-Manufacturing Center from the North Coast of Peru. Latin American Antiquity, 8 (1): 30-54.

FALBE, C.T.

1843 Vases antiques de Pérou. Copenhagen.

FORD, J.A.

1962 A quantitative method for deriving cultural chronology. Washington Pan American Union Technical Manual 1.

GEBAUER, A.B

1996 Pottery Production and the Introduction of Agriculture in Southern Scandinavia. W.K. Barnett; J.W. Hoopes (Eds.) The emergence of Pottery. Washington Smithsonian Institution Press: 99-114.

GIARDINO, M.; MILLER, R.; KUZIO,R.; MUIRHEAD, D.

1998 Analysis of Ceramic Color by Spectral Reflectance. American Antiquity, 63 (3): 477-484.

GIFFORD, J.C

1960 The type-variety method of ceramic classification as an indicator of cultural phenomena. American Antiquity, 25: 341-7.

GROEVIUS e GRONOVIUS

1694 Thesaurus antiquitatum, Traj. ad Rhenum.

GUTHE, C.E.

1927 A method of ceramic description. Michigan Papers of the Michigan Academy of Science, Arts and Letters 8.

HEGMON, M.; ALLISON, J.R.; NEFF, H.; GLASCOCK, M.D.

1997 Production of San Juan red ware in the northern southwest: insights into regional interaction in early Puebloan Prehistory. American Antiquity, 62 (3): 449-463.

HENDRICKSON, E.M.; Mc DONALD, M.A.

1983 Ceramic Form and Function: an Ethnographic Research and Archaeological application. America Anthropology, 85: 630-643.

HILL, J.N.

1970 Broken K Pueblo: prehistoric social organization in the American Southwest. Anthropological Papers of the Univ. of Arizona n. 18. Tucson Univ. of Arizona Press.

HIRTH, F.

1888 Ancient porcelain: a study in Chinese medieval industry and trade. Londres.

HODDER, I.

1982 Symbols in action: ethnoarchaeological studies in material culture. Cambridge, Cambridge Univ. Press.

1989 The meaning of things: material culture and symbolic expression. Boston, Unwin Human.

HOOPES, J.W

1995 Interaction in Hunting and Gathering Societies as a context for the emergence of pottery in the Central American Isthmus. W.K. Barnett; J.W. Hoopes (Eds.) The emergence of pottery. Washington, Smithsonian Inst. Press: 185-198.
JULIEN, S.

1856 Histoire de la fabrication de la porcelaine chinoise. Paris.

KENT, $S$.

1984 Method and theory for activity area research: an ethnoarchaeological approach. New York, Columbia Univ. Press.

KIDDER, A.V.

1924 An introduction to the study of Southwestern archaeology. Papers of the Southwestern Expedition, Department of Anthropology 1. New Haven Yale Univ. Press.

1931 The pottery of Pecos. Papers of the Southwestern Expedition, Department of Anthropology 5. New Haven, Yale Univ. Press.

KRAMER, C

1979 Ethnoarchaeology: implications to ethnography for archeology. New York, Columbia Univ. Press.

KRIEGER, A.D.

1944 The typological concept. American Antiquity, 9: 271-88.

LAYTON, R. (Ed.)

1994 Conflict in the archaeology of living traditions Londres, Roudledge.

LONGACRE, W.A. (Ed.)

1970 Archaeology as anthropology: a case study. Anthropological Papers of the University of Arizona, 17.

1991 Ceramic Ethnoarchaeology. Tucson, Univ. of Arizona Press.

MATSON, F.R.

1984 Ceramics and man reconsidered with some thoughts for the future. S.E.Van der Leeuw; A. C. Pritchard (Eds.) The many dimensions of pottery: ceramics in Archaeology and Anthropology. Cingula 7, Institute for Pre and Proto History. Amsterdam, University of Amsterdam: 25-49.

Mc GOVERN, P.E.; NOTIS, M.D. (Eds.)

1989 Cross-Craft and Cross-Cultural interactions in Ceramics. Ohio, The American Ceramic Society.

MOORE, A.M.T.

1995 The Inception of potting in Western Asia and Its Impact on Economy and Society. W.K. Barnett; J.W. Hoopes (Eds.) The emergence of Pottery - technology and innovation in ancient Societies. Washington, Smithsonian Institution Press: $39-54$

NEFF, H. (Ed.)

1992 Chemical Characterization of Ceramic Pastes in Archaeology. Monographs in World Archaeology n.7. Madison, Wisconsin Prehistory Press.

1993 Theory, sampling and analytical techniques in the Archaeological study of Prehistoric Ceramics. American Antiquity, 58: 23-44.

NELSON, N.C.

1916 Chronology of the Tano Ruins, American Anthropologist, New Mexico, 18 (2): 159-180. 
NORITANÉ, N.

1876-9 Notice historique sur les arts et les industries japonais. Paris.

ORTON, C.; TYERS, P.; VINCE, A.

1995 Pottery in Archaeology. Cambridge Manuals in Archaeology. Cambridge, Cambridge Univ. Press.

OSBORNE, $\mathbf{R}$.

1996 Pot, trade and archaic Greek economy. Antiquity, vol 70 n. 267: 31-44.

PÄRSSINEN, M.; SIIRIÄINEN, A.

1997 Inka-style ceramics and their chronological relationship to the Inka expansion in the Southern Lake Titicaca Area (Bolivia). Latin American Antiquity, 8 (3): 255-271.

PEACOCK, D.P.S.

1977 Ceramics in Roman and medieval archaeology. D.P.S. Peacock (Ed.) Pottery in early commerce. Londres, Academic Press: 21-34.

PETRIE, W.M.F

1891 Tell el Hesy (Lachish). Londres Palestine Exploration Society.

PLIQUE, A.E.

1887 Etude de céramique arverno-romaine. Caen.

POOL, C.A. \& BEY, G. (Ed.)

1992 Ceramic Production and Distribution: an integrated approach. Boulder, Colorado, Westview Press.

RHODES, $M$.

1979 Methods of cataloguing pottery in Inner London: as historical outline. Medieval Ceramics, Londres, 3: 81-108.

RICE, P.M.

1987 Pottery Analysis: a sourcebok. Chicago and London, The University of Chicago Press.

1988 Overview and Prospect. P.M. Rice (Ed.) Pots and Potters. Current Approaches in Ceramic Archaeology, Monograph XXIV, Inst. of Archaeology, Los Angeles Univ. of California: 245-255.

ROBINSON, A.M.

1979 Three approaches to the problem of pottery fabric descriptions. Medieval Ceramics, 3: 3-36.

ROBRAHN-GONZÁLEZ, E.M.

1996 Os grupos ceramistas pré-coloniais no CentroOeste Brasileiro. Rev. do Museu de Arqueologia e Etnologia, São Paulo 6: 83-121.

ROUSE, I.G.

1960 The classification of atifacts in archaeology. American Antiquity, 25: 313-23.

RYE, O.S.

1981 Pottery technology. Principles and reconstruction. Manuals on Archaeology 4, Washington DC, Smithsonian Inst. Press.

\section{SASSAMAN, K.E.}

1996 The social contradictions of Traditional and Innovative cooking Technologies in the Prehistoric American Southeast. W.K. Barnett; J.W. Hoopes (Eds.) The emergence of Pottery. Washington, Smithsonian Inst. Press: 223-240.
SCHOOLCRAFT, H.R.

1847 Notices of some antique earthen vessels of Florida. Nova Iorque.

SHEPARD, A.O.

1956 Ceramics for the Archaeologist. Washington Carnegie Institute of Washington.

1964 Temper identification: technological sherdsplitting or an unanswered challenge. American Antiquity, 29: 518-20.

SINOPOLI, C.M

1991 Approaches to Archaeological Ceramics. Nova

SKIBO, J. Iorque, Londres, Plenum Press.

1992 Pottery function: a use-alteratio perspective. Interdisciplinary Contributions to Archaeology. Nova Iorque, Londres, Plenum Press.

SKLENÁR, $K$.

1983 Archaeology in Central Europe: the first 500 years. Leicester, Leicester Univ. Press.

SMITH, C.R.

1854 Catalogue of the Museum of London antiquities collected by, and property of, Charles Roach Smith. Londres.

SOLON, M.L.

1910 Ceramic literature: an analytical index. Londres, Charles Griffin.

SPIER, L.

1917 An outline for a chronology of Zuñi ruins. Anthropological Papers of the American Museum of Natural Histoty, 18, Nova Iorque: 207-331.

SQUIER, E.G.; DAVIS, E.H.

1848 Ancient monuments of the Mississippi valley, Smithsonian Contributions to Knowledge I, Washington, Smithsonian Institution.

STOLTMAN, J.B.

1989 A Quantitative approach to the Petrographic analysis of Ceramic Thin Section. American Antiquity, 54: 157-160.

1991 Ceramic Petrography as a technique for documenting cultural interaction: an example from the Upper Mississippi Valley. American Antiquity, 56: 103-120.

STOW, J.

1603 A survey of London. London.

STRAZICICH, N.M.

1998 Clay sources, pottery production and regional economy in Chalchihuites, Mexico, AD 200900. Latin American Antiquity, 9 (3) September: $259-274$.

TSCHOPIK, H. Jr.

1950 An Andean ceramic tradition in historical perspective. American Antiquity, 15: 196-218.

VAN DER LEEUW, S.E.

1984 Dust to dust: a transformational view of the ceramic cycle. S.E.Van der Leew; A.C. Pritchard (Eds.) The many dimensions of pottery: ceramics in Archaeology and Anthropology Cingula 7, Inst. for Pre and Proto History. Amsterdam, Univ. of Amsterdam: 707-792.

VAN MELLEN, J.

1679 Historia urnae sepulchralis sarmaticae. Jena. 
VANDIVER, P.B.; SOFFER,O.; KLIMA,B.; SVOBODA, J.

1989 The origins of ceramic technology at Dolni Vestonice, Czechoslovakis. Science, 246: 10021008.

\section{WALDEK, F.}

1838 Voyage dans la province d'Yucat'n. Paris.

WHALLON, R. Jr.

1972 A new approach to pottery typology. American Antiquity, 37: 13-33.

WILLEY, G.; SABLOFF, J.

1974 A history of American Archaeology. Londres, Thames and Hudson.

Recebido para publicação em 11 de maio de 1998.
WÜST, I.

1990 Continuidade e mudança - para uma interpretação dos grupos ceramistas pré-coloniais da bacia do rio Vermelho, Mato Grosso. Tese de Doutoramento. São Paulo, FFLCH-USP.

YOUNG, W.J.; WHITMORE, F.E.

1980 Guidelines for the processing and publication of medieval pottery from excavations. Directorate of Ancient Monuments and Historic Buildings Occasional Paper 4, Londres, HMSO. 